\title{
Intercomparison of ADC-Lite Images on UAV and TM Simulation Data for Soybean Leaf Area Index Retrieval
}

\author{
Qi Zhang ${ }^{1,2,3,4}$, Zhongbin $\mathrm{Su}^{1 *}$, Guijun Yang ${ }^{2,3,4}$, Minghui Wang ${ }^{1}$, Weizheng \\ Shen ${ }^{1}$, Xiaowei Teng ${ }^{2,3,4}$ and Jinhui Dong ${ }^{2,3,4}$ \\ 1. College of Electronic Engineering and Information, Northeast Agricultural \\ University, Harbin 150030, China \\ 2. National Engineering Research Center for Information Technology in \\ Agriculture, Beijing 100097, China \\ 3. Key Laboratory of Agri-informatics, Ministry of Agriculture, Beijing \\ 100097, China \\ 4. Beijing Engineering Research Center of Agricultural Internet of Things, \\ Beijing 100097, China \\ Zhangqi920503@163.com
}

\begin{abstract}
Currently, TM images has a very high practical value and widely used in all aspects of agricultural. Unmanned Aerial Vehicles (UAV) remote sensing platform mounted ADC-Lite multi-spectral sensor has consistent channels response functions with TM sensor in TM2, TM3 and TM4, demonstrated to compete with TM sensor, due to low operational cost, high operational flexibility, high spatial resolution of imagery $(0.018 \mathrm{~m}$ with flight altitude $50 \mathrm{~m})$ and heterogeneity both at time and spatial-scale. In order to make sure whether it has widely used as TM sensor, moreover, the aim of this work is to assess ADC-Lite performance such as its adaptability and practicability. In this paper, ADC-Lite multi-spectral data, ground truth ASD hyperspectral and Leaf area index (LAI) data were acquired in soybean planting area, Jiaxiang County, Shandong Province on September18th, 2015. Since the ADC-Lite has different spatial scales with TM, this paper used TM simulation data transformed by ground truth ASD data, constructed LAI inversion model by empirical model based on two sensors and ground measured data, using 5 vegetation indices: ratio vegetation index (RVI), normalized difference vegetation index (NDVI), soil adjust vegetation index (SAVI), difference vegetation index (DVI) and triangular vegetation index (TVI). Determination coefficient $R^{2}$, root mean square error (RMSE) and the estimation accuracy $\left(E_{A}\right) 3$ indicators were acquired to assess the model. This work suggests that the established model of ADC-Lite sensor with TM simulation sensor has high consistency in accuracy. NDVI linear regression model derived from both of them presented a strong correlation with ground-measured LAI. It's preliminarily shown that ADC-Lite images assess soybean LAI is feasible. This is anticipated to have tremendous implications that $A D C$-Lite can be made supplement for existing satellites, aerial and ground sensing, provide important information for Crop condition monitoring and critical data to support crop maturity, nutrition monitoring and fertilization management.
\end{abstract}

Keywords: remote sensing, UAV, ADC-Lite, TM, LAI, soybean

\section{Introduction}

Leaf area index (LAI) is commonly defined as the sum of the unit surface area on one side of the blade [1]. It is a description of the most commonly used indicator of crop [2] and an important parameter for crop yield assessment [3-4]. Accurate estimates 
of the spatial variation of vegetation LAI and its phenology response which is anticipated to have tremendous implications for vegetation growth and photosynthesis, pest and disease monitoring, biomass estimates and carbon cycle [5].

Because of its rich resources, Satellite remote sensing can provide continuous planar information, has irreplaceable advantages in crop growth monitoring, yield estimates and other researches. Moreover, it has played an increasingly important role to guide agricultural production and management. Medium resolution satellite multi-spectral imaging is widely used in detection applications of crop growing for agricultural practical applications. In many of the medium resolution image data, the US Landsat TM series is more commonly used.

1990s, Thenkabail et al. [6] used TM images combined with the same period acquisition ground truth data to establish leaf area index estimation mode of soybeans and corns. The results showed that the prediction accuracy was up to 66 percent; In 2007, A supervised classification was carried to detect land-use changes by Adel Shalaby et al. [7] in the Northwestern coastal zone of Egypt from 1987 to 2001 for 14 years and study the reasons of land degradation by using TM images combined with GIS; In 2008, Bai Jie et al. [8] used three algorithms of surface temperature inversion for $\mathrm{TM} / \mathrm{ETM}^{+}$remote sensing data and validated the results with measured data, the results demonstrated single window algorithm had the best consistency with the ground truth data; In 2010, Zhang Jing Cheng et al. [9] based on HJ-CCD with TM images for rice LAI inversion, results showed high consistency of the two.

In recent years, the use of UAV technology has become a development tendency. Micro UAV remote sensing information acquisition technology with low running cost, high flexibility, efficient ways of accessing to data and images with high spatial resolution $(0.018 \mathrm{~m}$ with flight altitude $50 \mathrm{~m})$ has been widely used in the field of agriculture, making up Satellite remote sensing technology revisit long period, lack of image resolution and other shortcomings, is one of the main methods of rapid accessing to crops information and an important direction for the development of precision agriculture [10]. The use of UAV remote sensing platforms acquired GDNVI vegetation index of winter wheat with different nitrogen levels by Hunt ER et al. [11], which found it had a high correlation with LAI. Gao Lin et al. [12] obtained multi-spectral remote sensing image of soybean pod and seed filling stage through UAV equipped with ADC-Lite sensor, building the univariate and multivariate LAI inversion model and results showed that NDVI linear inversion model of seed filling stage of soybean worked best on LAI .The UAV platform designed for maize phenotyping studied had the potential to effectively assist in crop genetic improvement [13], providing a new feasible method for breeding.

In this paper, we present the use of multi-rotor UAV remote sensing platform mounted ADC-Lite sensor for flight monitoring about soybean planting area in Jiaxiang County Shandong Province, deriving multi-spectral remote sensing data and ground synchronized measured data such as ASD hyperspectral data and LAI data of this study area and transforming ASD hyperspectral data into TM multi-spectral sensor data. Five broad band vegetation indices were adopted to assess LAI retrieval accuracy with both sensors to establish empirical models which were aimed to analyze whether the ADC-Lite sensor could be as widely used as TM sensor, what's more, evaluate its feasibility and application of conducting agricultural monitoring, provide a theoretical basis and technical support for UAV remote sensing technology in the practical application of agricultural condition monitoring and a new method for the guidance of precision agriculture research. 


\section{Materials and Methods}

\subsection{Introduction of the Experiment Zone}

The experiment was carried out in test of Jiaxiang County, Jining City, Shandong Province, which is bounded both hilly area of central south of Shandong Province and the Huanghuaihai Plain in southwest China. The geographical scope of longitude is $116^{\circ} 22^{\prime} 10 " \sim 116^{\circ} 22 ' 20^{\prime \prime}$ and latitude is $35^{\circ} \sim 36^{\circ}$. It is mild and humid climate, four distinct seasons with adequate light, the average annual temperature of $13.9^{\circ} \mathrm{C}$, annual precipitation of $701.8 \mathrm{~mm}$ and the average frost-free period of 200 days. Wheat, rice and soybeans are the main crops of this area. Since good representation of this cultivation area such as the unique geography and climate, the structure of the soil and planting patterns, therefore, it is a good test area.

\subsection{Ground Data Acquisition}

The study area has a total of 46 cells ( 5 lines each cell, $5 \mathrm{~m}$ each line, $0.5 \mathrm{~m}$ line spacing). The use of LAI 2000C plant canopy analyzer measured Soybean LAI. Measurement time was 16: 00-18: 00 in the day of UAV sampling. When using the instrument, it should avoid direct sunlight, be close to the plant roots and keep the camera lens parallel to the ground, and then read five targets of each location. After taking the average for each location measurement results obtained soybeans LAI average value for each cell.

The Soybean canopy hyperspectral data were derived by the United States ASD Company FieldSpec Pro FR2500 hanging back field spectroradiometer (Analytical Spectral Devices). The wavelength range of the instrument is $350 \sim 2500 \mathrm{~nm}$, spectral resolution of $3 \mathrm{~nm}(350 \sim 1000 \mathrm{~nm})$ and $10 \mathrm{~nm}(1000 \sim 2500 \mathrm{~nm})$ and sampling interval $1 \mathrm{~nm}$. Measuring time range is GMT 10:30 14:30. Spectrometry need to select the cloudless, windless or small wind speed weather. When measuring, probe vertically downward, field of view angle was $25^{\circ}$. White calibration was required to reduce the impact of clouds and other changes to the spectral reflectance both before and after the observation target.

\subsection{TM Remote Sensing Data Acquisition and Processing}

TM sensor is a total of six channels in addition to thermal infrared. This study used three channels TM2, TM3 and TM4, whose band response functions were shown in Figure 1. ASD hyperspectral data were simulated to three bands of multiband reflectance corresponding to the measured TM spectrum. The way of simulating spectral response of each channel for satellite sensor is to use of the following equation:

$$
R i=\frac{\sum_{n=1}^{n} r(\lambda) \bullet \varphi i(\lambda)}{\sum_{n=1}^{n} \varphi i(\lambda)}
$$

$R i$ is the reflectance of band $i . \varphi i$ is spectral response function of wavelength $\lambda$ at band $i . r(\lambda)$ is the reflectance of wavelength $\lambda$. 


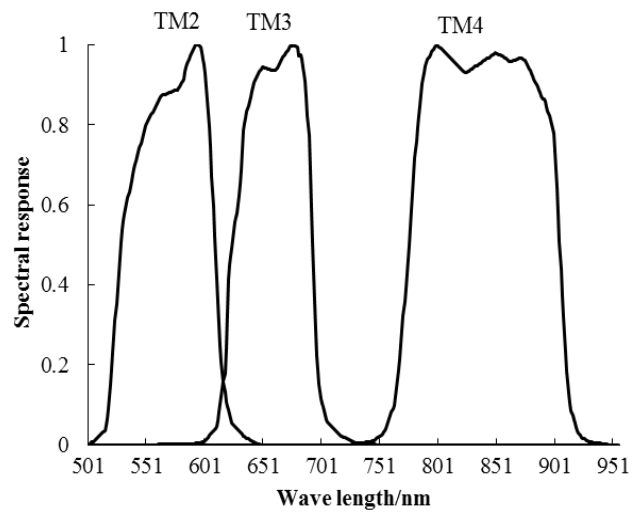

\section{Figure 1. Landsat TM-5 Satellite Sensors TM2, TM3, TM4 Spectral Response Functions}

\subsection{ADC-Lite Multi-Spectral Data Acquisition and Pre-Processing}

The test used eight-rotor electric UAV mounted ADC-Lite multi-spectral sensor to access multi-spectral images above altitude $50 \mathrm{~m}$. ADC-Lite multi-spectral sensor and TM sensor parameters are shown in Table 1. The original multi-spectral images were stitched by Pix4Dmapper developed of the Swiss Pix4D and multi-spectral image preprocessing included geometric correction which used history digital orthophoto image as a reference and radiometric calibration with pseudo-standard feature Radiation correct method. For geometric precision correction, the use of ENVI5.3 software to even choose 40 reference points of different positions of the image and then tested errors between two images by visual comparison of surface features (such as farm roads). By examining of this method, image geometric correction error was less than 0.5 pixels. For the radiometric calibration, white reflectivity average values of each band from $350 \mathrm{~nm} \sim 2500 \mathrm{~nm}$ bands were derived by processing white calibration cloth data of ASD spectrometer which used ViewSpecPro that is a kind of spectral data processing software. According to TM2, TM3, TM4 channel response functions, DN values of images were converted to multispectral image reflectance. Then the use of pretreated UAV multispectral images extracted the average vegetation indices of 46 cells.

Table 1. Fundamental Parameters of ADC-Lite and TM

\begin{tabular}{|c|c|c|c|}
\hline \multicolumn{2}{|c|}{ ADC-Lite multi-spectral sensor } & \multicolumn{2}{|c|}{ TM sensor } \\
\hline Parameter & Parameter value & Parameter & Parameter value \\
\hline Model & $\begin{array}{l}\text { Lightweight Agricultural } \\
\text { Digital Camera (ADC-Lite) }\end{array}$ & $\begin{array}{c}\text { Spatial } \\
\text { resolution }(\mathrm{m})\end{array}$ & $\begin{array}{c}\text { 30(band1 band5,band7) } \\
120 \text { (band 6) }\end{array}$ \\
\hline Pixel number & $2048 \times 1536$ & Breadth $(\mathrm{km})$ & 185 \\
\hline $\begin{array}{l}\text { Wavelength range } \\
(\mathrm{nm})\end{array}$ & 520 920nm(green, red, NIR) & $\begin{array}{l}\text { Revisiting period } \\
\text { (d) }\end{array}$ & 16 \\
\hline $\begin{array}{c}\text { Ground resolution } \\
(\mathrm{m})\end{array}$ & 0.018(flight altitude $50 \mathrm{~m}$ ) & & $450 \sim 515,525 \sim 605$ \\
\hline Band sensitivity & $\begin{array}{c}\text { Green,red,NIR sensitivity } \\
\text { with bands approximately } \\
\text { equal to TM2, TM3 and } \\
\text { TM4 }\end{array}$ & $\begin{array}{l}\text { Wavelength } \\
\text { range(nm) }\end{array}$ & $\begin{array}{c}630 \sim 690,775 \sim 900 \\
1550 \sim 1750,1550 \sim 1750 \\
1040 \sim 1250,2090 \sim 2350\end{array}$ \\
\hline
\end{tabular}




\section{Results and Discussion}

\subsection{Construction of Soybean LAI Inversion Model}

When using multi-spectral remote sensing image retrieval LAI, visible light (especially red band) and near-infrared reflectance combinations are usually adopted, herein by reference literature [14-18], this paper selects five kinds of broad band vegetation indices (Table 2) for the inversion of soybean LAI model.

Table 2. Formulas of the Vegetation Index for Retrieving Soybean

\begin{tabular}{ccc}
\hline vegetation index & Formula & Reference \\
\hline RVI & $\mathrm{RVI}=\rho_{\mathrm{NIR}} / \rho_{\mathrm{R}}$ & Pearson et al., 1972[13] \\
NDVI & $\mathrm{NDVI}=\left(\rho_{\mathrm{NIR}}-\rho_{\mathrm{R}}\right) /\left(\rho_{\mathrm{NIR}}+\rho_{\mathrm{R}}\right)$ & Rouse et al., $1974[14]$ \\
SAVI & $\mathrm{SAVI}=(1+\mathrm{L})\left(\rho_{\mathrm{NIR}}-\rho_{\mathrm{R}}\right) /\left(\rho_{\mathrm{NIR}}+\rho_{\mathrm{R}}+\mathrm{L}\right)$ & Huete., 1988[15] \\
DVI & $\mathrm{DVI}=\rho_{\mathrm{NIR}}-\rho_{\mathrm{R}}$ & Jordan., 1969[16] \\
TVI & $\mathrm{TVI}=60\left(\rho_{\mathrm{NIR}}-\rho_{\mathrm{G}}\right)-100\left(\rho_{\mathrm{R}}-\rho_{\mathrm{G}}\right)$ & Broge et al., 2001[17] \\
\hline
\end{tabular}

Note: $\rho_{\mathrm{NIR}}, \rho_{\mathrm{R}}, \rho_{\mathrm{G}}$ represent the near-infrared, infrared, green band reflectance respectively; $L$ represents soil adjustment factor, ranging from 0 to 1 , usually set to 0.5 .

Since chlorophyll content is decreased, red band reflectance is increased and near-infrared reflectance is decreased in soybean seed filling period, vegetation indices and LAI are highly correlated [19], therefore, we choose soybean seed filling period to conduct LAI inversion. In this study, regression modeling analysis between 46 measured LAI data of soybean seed filling period and the data were collected by two sensors respectively were established with exponential, linear, logarithmic and power four commonly used regression equations. To avoid the contingency of modeling, test samples were selected five groups, each group of 32 randomly selected sample of data as the modeling, and the remaining 14 as the test samples. LAI inversion modeling accuracy of the two sensors was compared and the estimation capacity of LAI was analyzed. Model calibration was used the coefficient of determination $\left(\mathrm{R}^{2}\right)$ and root mean square error (RMSE).

\subsection{LAI Inversion Model Accuracy Comparison and Analysis}

The study found that on the whole, the accuracy of univariate models with five vegetation indices based on ADC-Lite sensor and TM sensor was high and closed each other. All the models could reach a significant level of $P=0.01$ and the coefficient of determination $\left(\mathrm{R}^{2}\right)$ was 0.65 to 0.83 , root mean square error (RMSE) was 0.25 to 0.46 . On the basis of $\mathrm{R}^{2}$ and RMSE with comprehensive consideration, the best inversion models which were selected for each vegetation index based on ADC-Lite sensor and TM sensor are shown in Table 3. 
Table 3. Optimal Inversion of the Variable Models for Soybean LAI

\begin{tabular}{ccccc}
\hline Image type & Vegetation index & Model & $\mathrm{R}^{2}$ & RMSE \\
\hline \multirow{5}{*}{ ADC-Lite } & RVI & LAI $=1.3982 \times \ln (\mathrm{RVI})+0.6477$ & 0.777 & 0.369 \\
& NDVI & LAI $=4.4989 \times \mathrm{NDVI}+0.0876$ & 0.786 & 0.329 \\
& SAVI & LAI $=4.8836 \times$ SAVI +0.3461 & 0.763 & 0.373 \\
& DVI & LAI $=5.6973 \times \mathrm{DVI}+0.6159$ & 0.724 & 0.428 \\
& TVI & LAI $=0.0706 \times \mathrm{TVI}+0.6822$ & 0.733 & 0.422 \\
\hline \multirow{5}{*}{ TM } & RVI & LAI $=1.1104 \times \ln (\mathrm{x})+0.565$ & 0.825 & 0.364 \\
& NDVI & LAI $=4.3343 \times \mathrm{NDVI}-0.4205$ & 0.819 & 0.343 \\
& SAVI & LAI $=2.1405 \times \ln (\mathrm{SAVI})+4.0883$ & 0.801 & 0.410 \\
& DVI & LAI $=1.6766 \times \ln (\mathrm{DVI})+4.4672$ & 0.754 & 0.429 \\
& TVI & LAI $=1.5912 \times \ln (\mathrm{TVI})-2.2178$ & 0.759 & 0.452 \\
\hline
\end{tabular}

In groups of five tests, for ADC-Lite sensor, NDVI vegetation index had highest correlation with LAI, $\mathrm{R}^{2}$ and RMSE were higher than RVI. For TM sensor, RVI and NDVI vegetation indices were both reflected high correlation with LAI. $\mathrm{R}^{2}$ was slightly higher than RVI in NDVI model and RMSE was slightly lower than RVI, so the correlation analysis of best inversion models for RVI and NDVI based on TM sensor in Table 3 is shown in Figure 2. After the predicted values and measured values fitting, $R^{2}$ was 0.831 , RMSE was 0.347 for RVI and $\mathrm{R}^{2}$ was 0.851 , RMSE was 0.331 for NDVI.

It's visible that RVI fitting was not as effective as NDVI which was slightly better than RVI. With both sensors, NDVI vegetation index was seen most universal and had highest accuracy in LAI inversion. NDVI vegetation index by using a linear model of LAI inversion had highest precision, indicating the linear model was best suited for inversion LAI; throughout the study area, the linear model which was constructed based on NDVI vegetation index was the best for LAI inversion. Based on ADC-Lite and TM sensors, in 5 groups NDVI constructed model by utilizing the remaining 14 samples to verify the data had found that $\mathrm{R}^{2}$ was greater than 0.768 and 0.778 , RMSE was less than $0.329,0.343$ and estimation accuracy was greater than $86.2 \%, 86.4 \%$ respectively. It can be seen the linear model which was constructed based on NDVI had best universal and was suitable for LAI inversion in the entire region. Fitting between predicted values and measured values of NDVI linear inversion model based on the ADC-Lite sensor is shown in Figure 3. Comparative analysis of Figure 2 and Figure 3, it suggests that TM sensor worked best on LAI retrieval, $\mathrm{R}^{2}$ was 0.819 , RMSE was 0.343 and $\mathrm{E}_{\mathrm{A}}$ was $86.4 \%$; while the ADC-Lite sensor, $\mathrm{R}^{2}$ was 0.786 , RMSE was $0.329, \mathrm{E}_{\mathrm{A}}$ was $86.4 \%$. It can be seen that in the case without considering spatial scale TM, TM inversion was the best, but the ADC-Lite was very similar with it, results were consistent. It accounts for that ADC-Lite sensor could provide a theoretical basis and technical support for agricultural condition monitoring in practical applications and it is possible to provide a reliable guarantee for the precision agriculture research. 


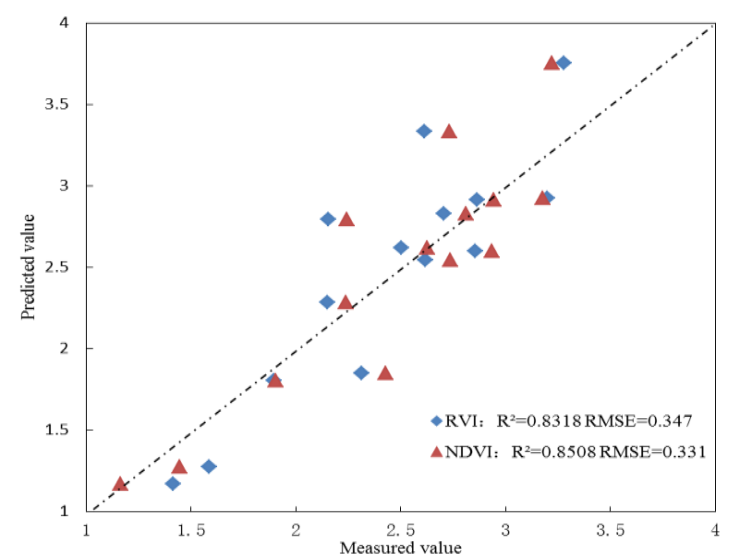

Figure 2. Comparison between Predicted and Measured Values of Two Retrieving Models Based On the TM Sensor

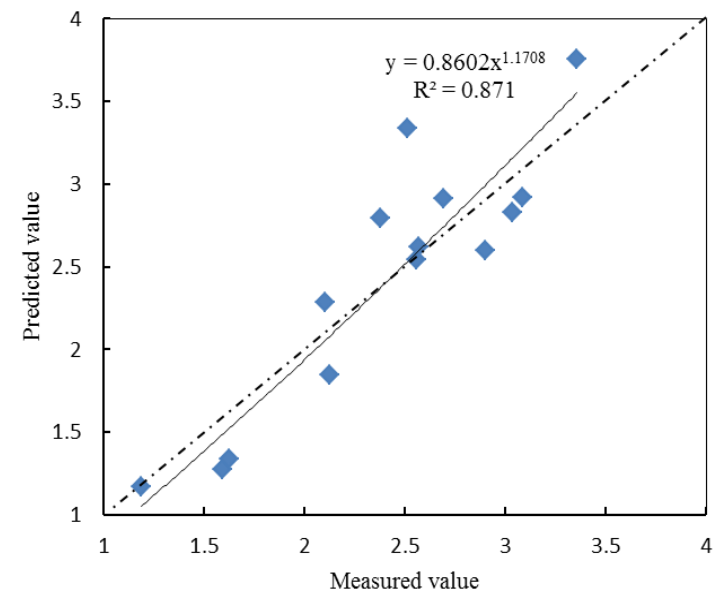

Figure 3. Comparison between Predicted Values and Measured Values Based on the ADC-Lite Sensor

\section{Conclusions}

In the field of agricultural remote sensing monitoring, the algorithms and models are generally applied to satellite image data [20-21], and the study that using the crop spectral information of Unmanned Aerial Vehicle (UAV) remote sensing to obtain crop physical parameters is still relatively small, so the contrast between the new sensor and some mature sensors has a profound significance to the agriculture monitoring with remote sensing information. The sensors which have similar band set, band response parameters should have a high consistency in spectral information. In this paper, the two kinds of sensors have a high consistency in the study of LAI inversion, which can confirm the conclusion to some extent.

Overall, the two kinds of sensors showed the high consistency in the process of LAI inversion, and the precision of the inversion models were high. This suggests that the LAI inversion accuracy of ADC-Lite sensor is close to TM sensor, and the ADC-Lite data has certain feasibility in crop condition monitoring. The TM data has a long cycle, and its accuracy is affected by the weather reason. But the atmosphere and clouds have less effect on low-altitude UAV remote sensing data, and the flight cycle can be adjusted according to the monitoring need, making continuous observation possible. 
The ADC-Lite low-altitude UAV remote sensing also has the advantage of low cost, high spatial resolution and so on. The analysis for the variation trend of spectral characteristics can provide crop monitoring important information and offer some decisions to field management. The further research should be carried out with more ADC-Lite data.

This study designed a UAV remote sensing platform, which mounted the ADC-Lite multispectral sensor. The soybean flight monitoring was executed with the UAV remote sensing platform in Jiaxiang county, Shandong province. Then the soybean LAI inversion results were analyzed and compared between ADC-Lite sensor and ASD simulated TM data. Main conclusions are as follows:

(1) The accuracy of the single variable model based on the ADC-Lite data and simulated TM data had high consistency.

(2) The NDVI linear regression model had the strongest ability of soybean LAI inversion, and the LAI inversion model (LAI=4.3343 $\times$ NDVI-0.4205) based on simulated TM data of the 5 groups samples had the best accuracy. The $\mathrm{R}^{2}$, RMSE and $\mathrm{E}_{\mathrm{A}}$ between measured LAI and simulated TM data inversion LAI were 0.819, 0.343 and $86.4 \%$ respectively. The $\mathrm{R}^{2}$, RMSE and $\mathrm{E}_{\mathrm{A}}$ between measured LAI and ADC-Lite data inversion LAI were $0.786,0.349$ and $86.5 \%$.

(3) The estimated LAI accuracies of soybean based on ADC-Lite data and simulated TM were similar. But the ADC-Lite low-altitude UAV remote sensing had the advantage of low cost, high spatial resolution and so on. The low-altitude UAV remote sensing was less affected by atmosphere and clouds. The convenience of flight monitoring became an important remote sensing data, and was more suitable for the need of precision agriculture.

(4) This research also had many shortcomings: this study built LAI inversion model had not considered other growth season factors; the number of measured samples and the robustness of the models were limited; The ASD simulated TM data had the same space scale with ADC-Lite data, but it existed differences with the true TM data.

\section{Acknowledgements}

The authors would like to acknowledge the financial support from the Key Technologies R\&D Program of Heilongjiang Province (GA15B106), National High Technology Research and Development Program (863 program), Miniature Unmanned Aircraft Remote Sensing Information Acquisition and Crop Nutrient Management Techniques (2013AA102303) and the Natural Science Fund Project of Beijing (4141001). The authors also wish to acknowledge the continuous support of the Northeast Agricultural University and National Engineering Research Center for Agricultural Information Technology.

\section{References}

[1] J. M. Chen and T. A, "Blank Defining leaf area index for non-flat leaves", Plant, Cell\& Environmentm vol. 15, no. 4, (1992), pp. 421-429.

[2] B. Yang and Z. Pei, "Definition of crop condition and crop monitoring using remote sensing", Transactions of the Chinese Society of Agricultural Engineering, vol. 15, no. 3, (1999), pp. 214-218.

[3] P. C. Doraiswamy, J. L. Hatfield, T. J. Jackson, B. Akhmedov, J. Prueger and A. Stern, "Crop condition and yield simulations using Landsat and MODIS", Remote Sensing of Environment, vol. 92, no. 4 , (2004), pp. 548-559.

[4] O. Abou-Ismail, J. F. Huang and R. C. Wang, "Rice yield estimation by integrating remote sensing with rice growth simulation model", Pedosphere, vol. 14, no. 4, (2004), pp. 519-526.

[5] W. Huang, M. Huang, L. Liu, J. Wang, C. Zhao and J. Wang, "Inversion of the severity of winter yellow rust using proper spectral index", Transactions of the Chinese Society of Agricultural Engineering, vol. 21, no.4, (2005), pp. 97-103

[6] P. S. Thenkabail, A. D. Ward and J. G. Lyon, "Landsat-5 Thematic Mapper models of soybean and corn crop characteristics", International Journal of Remote Sensing, vol. 15, no. 1, (1994), pp. 49-61. 
[7] A. Shalaby and R. Tateishi, "Remote sensing and GIS for mapping and monitoring land cover and land-use changes in the Northwestern coastal zone of Egypt", Applied Geography, vol. 27, no. 1, (2007), pp. 28-41.

[8] J. Bai, S. Liu and G. Hu, "Inversion and verification of land surface temperature with remote sensing $\mathrm{TM} / \mathrm{ETM}^{+}$data", Transactions of the Chinese Society of Agricultural Engineering, vol. 24, no.9, (2008), pp. 148-154.

[9] J. Zhang, X. Gu, J. Wang, W. Huang, X. He and H. Wang, "Analysis of consistency between HJ-CCD images and TM images in monitoring rice LAI", Transactions of the Chinese Society of Agricultural Engineering, vol. 26, no. 7, (2010), pp. 186-193.

[10] P. Wang, X. Luo, Z. Zhou, Y. Zang and S. Hu, "Key technology for remote sensing information acquisition based on micro UAV", Transactions of the Chinese Society of Agricultural Engineering, vol. 20, no.18, (2014), pp.1-12.

[11] R. E. Hunt, D.W. Hively, C. S. T. Daughtry and G. W. McCary, "Remote Sensing of Crop Leaf Area Index Using Unmanned Airborne Vehicles", ASPRS Pecora 17 Conference Proceeding, American Society for Photogrammetry and Remote Sensing, Bethesda, MD. Vol. 12, (2008).

[12] L. Gao, G. Yang, B. Wang, H. Yu, B. Xu and H. Feng, "Soybean leaf area index retrieval with UAV (unmanned aerial vehicle) remote sensing imagery", Chinese Journal of Eco-Agriculture, vol. 23, no. 7, (2015), pp. 868-876.

[13] M. Zaman-Allah, O. Vergara, J. L. A. Araus Tarekegne, C. Magorokosho, P. .J Zarco-Tejada and A. Hornero, "Unmanned aerial platform-based multi-spectral imaging for field phenotyping of maize", Plant Methods. Vol. 11, no. 1, (2015), pp. 1.

[14] R. L. Pearson and L. D. Miller, "Remote mapping of standing crop biomass for estimation of the productivity of the short grass prairie", Proceeding of the 8th International Symposium on Remote Sensing of the Environment. Michigan, Ann Arbor, Michigan USA, vol. 1, (1972), pp. 1355-1379.

[15] J. W. Rouse, R. H. Haas, J. A. Schell and D. W. Deering, "Monitoring vegetation systems in the Great Plains with ERTS”, NASA Special Publication, vol. 351, (1974), pp. 309-313.

[16] A R. A. Huete, "soil-adjusted vegetation index (SAVI)", Remote Sensing of Environment, vol. 25, no. 3, (1988), pp. 295-309

[17] C. F. Jordan, "Derivation of leaf-area index from quality of light on the forest floor", Ecology, vol. 50, no. 4 , (1969), pp. 663-666

[18] N. H. Broge and E. Leblan, "Comparing prediction power and stability of broadband and hyperspectral vegetation indices for estimation of green leaf area index and canopy chlorophyll density", Remote Sensing of Environment, vol. 76, no.2, (2001), pp. 156-172.

[19] G. D. Badhwar, "Crop emergence date determination from spectral data", Photogrammetric Engineering and Remote Sensing, vol. 46, no. 3, (1980), pp. 369-377.

[20] F. Yan, B. Wu and Y. Wang, "Estimating spatiotemporal patterns of aboveground biomass using Landsat TM and MODIS images in the Mu Us Sandy Land, China”, Agricultural and Forest Meteorology, vol. 200, (2015), pp. 119-128

[21] L. Wang, G. Zheng, H. Chen, C. Zou, Z. Liu and Y. Cheng, "Monitoring the change of global winter wheat planting area in Henan province based on HJ-CCD image", Chinese Journal of Agricultural resources and regional planning, vol. 32, no. 2, (2011), pp. 58-62.

\section{Authors}

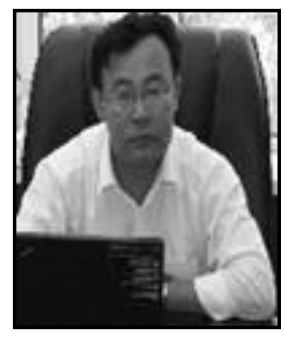

Zhongbin Su (1965-), he is male, Ph.D. professor, president of the college of electrical and information in Northeast Agricultural University, mainly engaged in the research and application of information technology in agriculture. 
International Journal of Hybrid Information Technology Vol. 9, No.7 (2016) 